\title{
Kramers equation for a charged Brownian particle: The exact solution
}

\author{
Tania P. Simões ${ }^{a)}$ and Roberto E. Lagos ${ }^{b), *}$ \\ a) Instituto de Física 'Gleb Wataghin' Universidade Estadual de Campinas \\ (UNICAMP) CP. 6165, 13083 - 970 Campinas, SP Brazil. \\ b) Departamento de Física, IGCE Universidade Estadual Paulista \\ (UNESP) CP. 178, 13500-970 Rio Claro, SP, Brazil. \\ * Virtual Research Center-CC@Complex-Science and Computing \\ for Complexity (UNESP SP Brazil)
}

\begin{abstract}
We report the exact fundamental solution for Kramers equation associated to a brownian gas of charged particles, under the influence of homogeneous (spatially uniform) otherwise arbitrary, external mechanical, electrical and magnetic fields. Some applications are presented, namely the hydrothermodynamical picture for Brownian motion in the long time regime.
\end{abstract}

\section{INTRODUCTION}

In Chandrasekhar's 1943 celebrated paper ${ }^{1}$, Kramers equation $^{2}$ was solved for the free Brownian particle and some general lines were drawn towards solving this problem in a field of force. Only recently some progress was reported considering Kramers equation for a charged Brownian particle in a field of force: Czopnik \& Garbaczewsky $(\mathrm{CG})^{3}$ solved Kramers planar equation in a magnetic field, essentially transforming the magnetic field contribution into a tensorial Stokes-like dissipative force. Later, Ferrari ${ }^{4}$ via transformed phase space variables, mapped Kramers equation for a charged Brownian particle in an electric field into the free Brownian particle case. By combining both CG's 'rotated' Stokes force and Ferrari's gauge, in section II we report the exact fundamental solution of Kramers equation for a charged Brownian particle in an uniform, otherwise arbitrary field of forces. In section III we present some applications, concerning the hydrothermodynamical picture of Brownian motion, the validity of the local equilibrium approximation and the 'linear' regime (see for example ${ }^{5,6}$ ). Comparison is made with some results obtained via a perturbative recursive scheme ${ }^{7,8}$. Finally in section IV we present some concluding remarks and outline some work in progress.

\section{FUNDAMENTAL SOLUTION}

We study a Brownian gas composed of charged particles (mass $m$, charge $e$ ) under the influence of external fields: mechanic (mec), electric (E) and magnetic (B) fields, uniform in space and in general time dependent. Our starting point is Kramers equation for the density probability distribution $P(\mathbf{x}, \mathbf{v}, t)$ in phase space (position $\mathbf{r}$, velocity $\mathbf{v}$ ) at time $t$, in contact with a reservoir at temperature $T_{R}$ and under the force fields

$$
\mathbf{F}(\mathbf{v}, t)=\mathbf{F}_{m e c}+e \mathbf{E}+\frac{e}{c} \mathbf{v} \times \mathbf{B}
$$

The associated Kramers equation ${ }^{2}$ reads

$$
\frac{\partial P}{\partial t}+\mathbf{v} \frac{\partial P}{\partial \mathbf{x}}+\frac{\mathbf{F}}{m} \frac{\partial P}{\partial \mathbf{v}}=-\frac{\partial}{\partial \mathbf{v}} \frac{\mathbf{F}_{d}}{m} P+\lambda \frac{T_{R}}{m} \frac{\partial^{2} P}{\partial \mathbf{v}^{2}}
$$

with Boltzmann's constant $k_{B} \equiv 1, \lambda=\tau^{-1}$ is the friction coefficient (inverse of the collision time) and $\mathbf{F}_{d}=-\lambda m \mathbf{v}$ is the dissipative Stokes-like force. As in previous work $^{7,8}$, where a perturbative recursive scheme was presented for a more general case, we define $v_{T}$, a thermal velocity given by $m v_{T}^{2}=T_{R}$ and dimensionless variables by scaling space, velocity and time, respectively with $l=\tau v_{T}, v_{T}$ and $\tau$. Also we define the conservative acceleration a (and the associated potential $\phi$ ) and the cyclotronic frequency vector $\boldsymbol{\Omega}$ respectively as (hereafter all quantities are dimensionless unless stated otherwise)

$$
\begin{aligned}
\mathbf{a} & =\frac{\tau}{m v_{T}}\left(\mathbf{F}_{m e c}+e \mathbf{E}\right)=-\frac{\partial \phi}{\partial \mathbf{x}} \\
\boldsymbol{\Omega} & =\frac{e \tau}{m c} \mathbf{B}=\omega \hat{\omega}
\end{aligned}
$$

Notice that in terms of the usual dimensional cyclotronic frequency $^{8}$, we have $\omega=\omega_{c} \tau$. Concerning notation, we chose the very convenient bra-ket convention. Denote any vector $\mathbf{V}$ by $\mathbf{V}=V_{x}|x\rangle+V_{y}|y\rangle+V_{z}|z\rangle$ and its adjoint by $\mathbf{V}^{\dagger}=V_{x}\langle x|+V_{y}\langle y|+V_{z}\langle z|$ (all quantities are real). We also define some useful dyadics, namely: $\mathbf{e}_{1}=|z\rangle\langle z|$, $\mathbf{e}_{2}=|x\rangle\langle x|+| y\rangle\left\langle y\left|, \mathbf{e}_{3}=\right| x\right\rangle\langle y|-| y\rangle\langle x|$ and the unit dyadic $\mathbf{e}=\mathbf{e}_{1}+\mathbf{e}_{2}$. Furthermore, we define the $\mathrm{z}$-axis as the magnetic field direction $(\hat{\omega}=\hat{z})$, and Kramers equation (2) is cast in a compact form as

$$
\frac{\partial P}{\partial t}+\mathbf{v} \frac{\partial P}{\partial \mathbf{x}}+\mathbf{a} \frac{\partial P}{\partial \mathbf{v}}=\frac{\partial}{\partial \mathbf{v}} \boldsymbol{\Lambda} \mathbf{v} P+\frac{\partial^{2} P}{\partial \mathbf{v}^{2}}
$$

where the magnetic contribution is included as a tensorial Stokes-like dissipative term $\left(\mathrm{see}^{3,8}\right)$ 


$$
\mathbf{\Lambda} \mathbf{v}=\mathbf{v}+\mathbf{\Omega} \times \mathbf{v}=\left(\mathbf{e}-\omega \mathbf{e}_{3}\right) \mathbf{v}
$$

We also define

$$
\mathbf{M}=\mathbf{\Lambda}^{-1}=\mathbf{e}_{1}+\alpha\left(\mathbf{e}_{2}+\omega \mathbf{e}_{3}\right)
$$

with $\alpha^{-1}=1+\omega^{2}$. In dimensional form this tensorial collision time constant has the familiar form ${ }^{8}$

$$
\mathbf{M}=\frac{\tau}{1+\left(\omega_{c} \tau\right)^{2}}\left(\begin{array}{ccc}
1 & \omega_{c} \tau & 0 \\
-\omega_{c} \tau & 1 & 0 \\
0 & 0 & 1+\left(\omega_{c} \tau\right)^{2}
\end{array}\right)
$$

Since the planar $(x, y)$ dynamics is decoupled from the $z$ axis dynamics, the case $\mathbf{a} \equiv \mathbf{0}$ can be trivially solved generalizing the planar results of $(\mathrm{CG})^{3}$. Then, transforming to new variables $\mathbf{R}$ and $\mathbf{V}$, via Ferrari's gauge ${ }^{4}$ we map our problem to the free Brownian particle solved in 1943 by Chandrasekhar ${ }^{1}$. Here we particularize to time independent external fields case, yielding Ferrari's transformed variables ${ }^{4}$

$$
\begin{aligned}
& \mathbf{R}=\mathbf{x}-\delta \mathbf{x}(\mathbf{a}, \mathbf{M}, t) \\
& \mathbf{V}=\mathbf{v}-\delta \mathbf{v}(\mathbf{a}, \mathbf{M}, t)
\end{aligned}
$$

where

$$
\begin{gathered}
\delta \mathbf{v}=\mathbf{M}(\mathbf{1}-\boldsymbol{\Theta}) \mathbf{a}+\boldsymbol{\Theta} \mathbf{v}_{0} \\
\delta \mathbf{x}=\mathbf{M a} t-\mathbf{M}^{2}(1-\boldsymbol{\Theta}) \mathbf{a}+\mathbf{M}(1-\boldsymbol{\Theta}) \mathbf{v}_{0}+\mathbf{x}_{0} \\
\quad \boldsymbol{\Theta}=\exp (-t)\left(\mathbf{e}_{1}+\mathbf{e}_{2} \cos \omega t+\mathbf{e}_{3} \sin \omega t\right)
\end{gathered}
$$

and with Kramers equation (5) mapped to the trivially solved $^{3,4,1}$ equation

$$
\frac{\partial P}{\partial t}+\mathbf{V} \frac{\partial P}{\partial \mathbf{R}}=\frac{\partial}{\partial \mathbf{V}} \mathbf{\Lambda} \mathbf{V} P+\frac{\partial^{2} P}{\partial \mathbf{V}^{2}}
$$

The fundamental solution $G\left(\mathbf{x}, \mathbf{t}, t \mid \mathbf{x}_{0}, \mathbf{v}_{0}\right)$, namely with free boundary conditions and the point-like initial condition

$$
G\left(\mathbf{x}, \mathbf{v}, t=0 \mid \mathbf{x}_{0}, \mathbf{v}_{0}\right)=\delta\left(\mathbf{x}-\mathbf{x}_{0}\right) \delta\left(\mathbf{v}-\mathbf{v}_{0}\right)
$$

is given by

$$
G\left(\mathbf{x}, \mathbf{t}, t \mid \mathbf{x}_{0}, \mathbf{v}_{0}\right)=\left(\frac{1}{2 \pi}\right)^{3} \frac{1}{\Delta \sqrt{\Delta^{*}}} \exp -\frac{1}{2} \boldsymbol{\Phi}
$$

where

$$
\boldsymbol{\Phi}=\boldsymbol{\Phi}_{d}-\boldsymbol{\Phi}_{s}-\boldsymbol{\Phi}_{a}
$$

with

$$
\begin{aligned}
\boldsymbol{\Phi}_{d} & =\mathbf{V}^{\dagger} \mathbf{A}_{v} \mathbf{V}+\mathbf{R}^{\dagger} \mathbf{A}_{r} \mathbf{R} \\
\boldsymbol{\Phi}_{s n} & =\mathbf{V}^{\dagger} \mathbf{A}_{m} \mathbf{R}+\mathbf{R}^{\dagger} \mathbf{A}_{m} \mathbf{V} \\
\boldsymbol{\Phi}_{a} & =2 \mathbf{Q}^{\dagger}(\mathbf{R} \times \mathbf{V})
\end{aligned}
$$

or in a compact form

$$
\mathbf{\Phi}=\left(\begin{array}{ll}
\mathbf{V}^{\dagger} & \mathbf{R}^{\dagger}
\end{array}\right) \mathbf{A}\left(\begin{array}{l}
\mathbf{V} \\
\mathbf{R}
\end{array}\right)
$$

where

$$
\mathbf{A}=\left(\begin{array}{cc}
\mathbf{A}_{v} & -\mathbf{A}_{m}+|\mathbf{Q}| \mathbf{e}_{3} \\
-\mathbf{A}_{m}-|\mathbf{Q}| \mathbf{e}_{3} & \mathbf{A}_{r}
\end{array}\right)
$$

with

$$
\begin{aligned}
\mathbf{A}_{\alpha}= & \frac{a_{\alpha}}{\Delta} \mathbf{e}_{2}+\frac{a_{\alpha}^{*}}{\Delta^{*}} \mathbf{e}_{1} \\
\mathbf{Q} & =\frac{k}{\Delta} \hat{\omega} \\
\Delta & =a_{v} a_{r}-a_{m}^{2}-k^{2}
\end{aligned}
$$

and where

$$
a_{r}=1-b_{e}^{2}
$$

$$
a_{m}=\alpha\left(1-2 b_{e} b_{c}+b_{e}^{2}\right)
$$

$$
k=\alpha\left(2 b_{e} b_{s}-\omega a_{r}\right)
$$

$$
\begin{gathered}
a_{v}=\alpha\left(a_{r}+2 t-4 \alpha\left(1+b_{e}\left(\omega b_{s}-b_{c}\right)\right)\right) \\
b_{e}=\exp (-t), \quad b_{c}=\cos \omega t, \quad b_{s}=\sin \omega t
\end{gathered}
$$

The superscript $*$ in equations $(7,8)$ denotes the corresponding quantity evaluated at null magnetic field $(\omega \equiv$ $0)$.

The general solution satisfying the initial condition $P(\mathbf{x}, \mathbf{v}, t=0)=P_{0}(\mathbf{x}, \mathbf{v})$ is given by

$$
P(\mathbf{x}, \mathbf{v}, t)=\int d \mathbf{x}_{0} d \mathbf{v}_{0} G\left(\mathbf{x}, \mathbf{v}, t \mid \mathbf{x}_{0}, \mathbf{v}_{0}\right) P_{0}\left(\mathbf{x}_{0}, \mathbf{v}_{0}\right)
$$

We normalize the probability $P$ to $N$, the total number of particles in the gas and define the particle density

$$
n(\mathbf{x}, t)=\int d \mathbf{v} P(\mathbf{x}, \mathbf{v}, t)
$$

requiring

$$
N=\int d \mathbf{x} d \mathbf{v} P_{0}(\mathbf{x}, \mathbf{v})=\int d \mathbf{x} d \mathbf{v} P(\mathbf{x}, \mathbf{v}, t)
$$

A mean density $n_{0}$ is defined in the thermodynamic limit as $N=n_{0} V$ where the volume is defined by $V=\int d \mathbf{x}$. Thermal equilibrium conditions (TEC) are reached in the asymptotic regime $t \rightarrow \infty$ under null external fields, where we retrieve the Maxwellian distribution

$$
P_{T E C}(\mathbf{v})=(2 \pi)^{-3 / 2} n_{0} \exp -\frac{1}{2} \mathbf{v}^{2}
$$




\section{HYDROTHERMODYNAMICS OF BROWNIAN MOTION}

We start by defining some quantities of interest ${ }^{7,8}$ : particle flow density $\mathbf{J}$, the associated hydrodynamic velocity $\mathbf{u}$, kinetic energy density $\varepsilon$, local gas temperature $\theta$ (in $T_{R}$ units), local pressure $p$, entropy density $s$, Gibbs energy density $g$ and the total chemical potential $\mu$, respectively defined by

$$
\begin{gathered}
\mathbf{J}(\mathbf{x}, t)=\int d \mathbf{v} \mathbf{v} P(\mathbf{x}, \mathbf{v}, t)=n(\mathbf{x}, t) \mathbf{u}(\mathbf{x}, t) \\
\varepsilon(\mathbf{x}, t)=\frac{3}{2} n(\mathbf{x}, t) \theta(\mathbf{x}, t)=\frac{1}{2} \int d \mathbf{v} \mathbf{v}^{2} P(\mathbf{x}, \mathbf{v}, t) \\
p(\mathbf{x}, t)=n(\mathbf{x}, t) \theta(\mathbf{x}, t) \\
s(\mathbf{x}, t)=-\int d \mathbf{v} P(\mathbf{x}, \mathbf{v}, t) \ln \varkappa P(\mathbf{x}, \mathbf{v}, t) \\
g(\mathbf{x}, t)=\varepsilon(\mathbf{x}, t)-\theta(\mathbf{x}, t) s(\mathbf{x}, t)+p(\mathbf{x}, t) \\
\mu(\mathbf{x}, t)=\frac{g(\mathbf{x}, t)}{n(\mathbf{x}, t)}+\phi
\end{gathered}
$$

The additive constant

$$
\ln \varkappa=-1+3 \ln \frac{h}{\tau T_{R}}
$$

is chosen such that under TEC we retrieve the usual thermodynamical entropy density ${ }^{7,8}$. This equilibrium entropy and the associated chemical potential are respectively given by (in dimensional form)

$$
\begin{gathered}
s_{e q}\left(n_{0}, T_{R}\right)=n_{0}\left(\frac{5}{2}+\ln \frac{n_{Q}\left(T_{R}\right)}{n_{0}}\right) \\
\mu_{e q}\left(n_{0}, T_{R}\right)=-T_{R} \ln \frac{n_{Q}\left(T_{R}\right)}{n_{0}}
\end{gathered}
$$

with

$$
n_{Q}\left(T_{R}\right)=\left(\frac{2 \pi m T_{R}}{h^{2}}\right)^{\frac{3}{2}}
$$

Furthermore we define an entropy flux density as

$$
\mathbf{J}_{s}=-\int d \mathbf{v} \mathbf{v} P(\mathbf{x}, \mathbf{v}, t) \ln \varkappa P(\mathbf{x}, \mathbf{v}, t)
$$

Balance equations are computed as in ${ }^{7,8}$, yielding the continuity (Smoluchowsky) and the entropy balance equations, given respectively by

$$
\frac{\partial n}{\partial t}+\nabla \mathbf{J}=0
$$

and

$$
\frac{\partial s(\mathbf{x}, t)}{\partial t}+\frac{\partial \mathbf{J}_{s}(\mathbf{x}, t)}{\partial \mathbf{x}}=\sigma(\mathbf{x}, t)
$$

where the entropy production density $\sigma(\mathbf{x}, t)$ is given by

$$
\sigma(\mathbf{x}, t)=\int d \mathbf{v} P(\mathbf{x}, \mathbf{v}, t)\left(\frac{\partial \ln P(\mathbf{x}, \mathbf{v}, t)}{\partial \mathbf{v}}\right)^{2}-3 n(\mathbf{x}, t)
$$

In this brief report we compute the non equilibrium long time regime (LTR, the limit $t \gg 1$ and with non zero external fields). In this limit we have:

$$
\begin{aligned}
& \delta \mathbf{v}=-\mathbf{M} \frac{\partial \phi}{\partial \mathbf{x}}=\mathbf{V}_{F} \\
& \delta \mathbf{x}=\mathbf{V}_{F} t+\mathbf{x}^{*} \quad \mathbf{x}^{*}=\mathbf{x}_{0}-\mathbf{M}\left(\mathbf{V}_{F}-\mathbf{v}_{0}\right)
\end{aligned}
$$

Thus, in the LTR Ferrari's ${ }^{4}$ transformation is simply represented by the constant velocity shift $\mathbf{V}_{F}$ or equivalently, given by the solution of the (dimensional) equation

$$
\frac{\mathbf{V}_{F}}{\tau}=\frac{1}{m}\left(\mathbf{F}_{m e c}+e \mathbf{E}+\frac{e}{c} \mathbf{V}_{F} \times \mathbf{B}\right)
$$

We highlight some results in the LTR, corroborating some previously obtained results, via a recursive $\operatorname{method}^{7,8}$. We may cast

$$
P(\mathbf{x}, \mathbf{v}, t)=n(\mathbf{x}, t) W(\mathbf{x}, \mathbf{v}, t)
$$

where to lowest order in $t^{-1}$ we have

$$
\begin{gathered}
W(\mathbf{x}, \mathbf{v}, t)=\left(\frac{1+2 t}{4 \pi t}\right)^{\frac{3}{2}} \exp -\frac{(1+2 t)}{4 t}\left(\mathbf{V}-\mathbf{V}_{D}\right)^{2} \\
n(\mathbf{x}, t)=\left(\frac{1}{4 \pi t}\right)^{\frac{3}{2}} \frac{1}{\alpha} \exp -\frac{1}{2} \Gamma(\mathbf{R})
\end{gathered}
$$

with

$$
\Gamma(\mathbf{R})=\frac{1}{2 \alpha t}\left(\mathbf{R}^{2}+\alpha(\boldsymbol{\Omega R})^{2}\right)-\mathbf{V}_{D}^{2}
$$

$$
\mathbf{V}_{D}=\frac{1}{2 t}(\mathbf{R}+\mathbf{R} \times \mathbf{\Omega})
$$

Then it is also satisfied the relation

$$
\mathbf{J}(\mathbf{x}, t)=-\mathbf{M}\left(\frac{\partial n}{\partial \mathbf{x}}+n \frac{\partial \phi}{\partial \mathbf{x}}\right)
$$

We define a magneto covariant derivative as in ${ }^{8}$ for any given function $f(\mathbf{x}, t)$ as (notice the potential $\phi$ is expressed in $T_{R}$ units) 


$$
\begin{aligned}
D_{x} f & =\mathbf{M} \exp (-\phi) \frac{\partial}{\partial \mathbf{x}} f(\mathbf{x}, t) \exp (\phi) \\
& =\mathbf{M}\left(\frac{\partial f(\mathbf{x}, t)}{\partial \mathbf{x}}+f(\mathbf{x}, t) \frac{\partial \phi}{\partial \mathbf{x}}\right)
\end{aligned}
$$

and from (10) and (11) we retrieve Smoluchowsky equation with a magnetic field ${ }^{8}$

$$
\frac{\partial n}{\partial t}=\frac{\partial}{\partial \mathbf{x}}\left(D_{x} n\right)
$$

Also, the local temperature $T(\mathbf{x}, t)$, the entropy density and the total chemical potential (in dimensional form) are given by the following expressions

$$
\begin{gathered}
\frac{T(\mathbf{x}, t)}{T_{R}}=\theta(\mathbf{x}, t)=1-\frac{1}{2 t}+\frac{1}{3} \mathbf{u}^{2}(\mathbf{x}, t) \\
s(\mathbf{x}, t)=s_{e q}(n(\mathbf{x}, t), T(\mathbf{x}, t))+\delta s(\mathbf{x}, t) \\
\mu(\mathbf{x}, t)=\mu_{e q}(n(\mathbf{x}, t), T(\mathbf{x}, t))+\delta \mu(\mathbf{x}, t)
\end{gathered}
$$

where the entropy and chemical potential shifts, are respectively given by (to lowest order in $t^{-1}$ and $\mathbf{u}^{2} \sim \mathbf{M}^{2}$ )

$$
\begin{gathered}
\delta s(\mathbf{x}, t)=-\frac{1}{2} n(\mathbf{x}, t) \mathbf{u}^{2}(\mathbf{x}, t) \\
\delta \mu(\mathbf{x}, t)=\frac{1}{2} T(\mathbf{x}, t) \mathbf{u}^{2}(\mathbf{x}, t)+\phi(\mathbf{x}, t)
\end{gathered}
$$

Furthermore, the hydrodynamic velocity $\mathbf{u}$ may be cast in several equivalent forms, namely

$$
\mathbf{u}(\mathbf{x}, t)=D_{x} \ln n=\mathbf{M} \frac{\partial}{\partial \mathbf{x}}\left(\frac{\mu(\mathbf{x}, t)}{\theta(\mathbf{x}, t)}\right)=\mathbf{V}_{D}+\mathbf{V}_{F}
$$

Lat us briefly comment on the last equation. The entropy density (equation 14) with $\mathbf{u}(\mathbf{x}, t)=D_{x} \ln n$ is formally reminiscent of a Ginzburg-Landau expansion as noted in $^{8}$. Also, as stated by Landauer ${ }^{9}$ the gradient of a bona fide nonequilbrium chemical must be proportional to the particle flux $\mathbf{J}=n \mathbf{u}$.

It was found ${ }^{8}$ that a suitable expansion parameter is the collision time $\tau$ (or its tensorial partner $\mathbf{M}$ ). In the LTR and confirming our conclusions in $^{8}$, for the Brownian motion of a charged particle, the local equilibrium hypothesis is satisfied only to first order (linear) in $\tau(\mathbf{M})$ and where, among other things, Onsager relations are satisfied. In this linear approximation we have $T(\mathbf{x}, t) \approx T_{R}, \delta s(\mathbf{x}, t) \approx 0, \delta \mu(\mathbf{x}, t) \approx \phi(\mathbf{x}, t)$. Furthermore up to second order in $\tau$

$$
\mathbf{J}(\mathbf{x}, t) \approx \frac{1}{2} \frac{\partial \phi}{\partial \mathbf{x}} \quad \sigma(\mathbf{x}, t) \approx \frac{3}{2 t} n(\mathbf{x}, t)
$$

yielding a nonequilibrium temperature independent of the magnetic field, and a positive definite entropy production that vanishes as $t^{-1}$.

\section{CONCLUDING REMARKS}

Here we have presented the fundamental exact solution for Kramers equation in a field of uniform forces, hitherto unknown, and applied the results to the long time regime. Work in progress seeks solutions for general initial conditions and other than free boundary conditions (for example membranes ${ }^{10}$ ) and the associated hydrothermodynamical picture, to be inserted into a more general framework ${ }^{5,6}$. Also, we will address the question of a nonuniform reservoir temperature $T_{R}(\mathbf{x})^{8,11}$ and the inclusion of chemical reactions ${ }^{8,12}$ to our Brownian scheme.

Acknowledgment: This work was partially supported by $\mathrm{CNPq}$ (Brasil), and submitted in partial fulfillment of the requirements for the doctoral degree (TPS).

${ }^{1}$ S. Chandrasekhar, Rev. Mod. Phys. 151 (1943).

${ }^{2}$ H. A. Kramers, Physica 7284 (1940).

${ }^{3}$ R. Czopnik \& P. Garbaczewsky, Phys. Rev. E 63021105 (2001).

${ }^{4}$ L. Ferrari, J. Chem. Phys. 11811092 (2003).

${ }^{5}$ D. Jou, J. Casas-Vásquez and G. Lebon, Rep. Prog. Phys. 621035 (1999), ibid. 511105 (1988).

${ }^{6}$ J. M. G. Villar and J. M. Rubí, Proc. Natl. Acad. Sci. 98 11081 (2001)

${ }^{7}$ L. A. Barreiro, J. R. Campanha \& R. E. Lagos, Physica A 283160 (2000) (See also arXiv: cond-mat/9910405).

${ }^{8}$ L. A. Barreiro, J. R. Campanha \& R. E. Lagos,Rev. Mex. Fís. 4813 (2002) (See also arXiv:cond-mat/0012187).

${ }^{9}$ R. Landauer, Helv. Phys. Acta 56847 (1983).

10 T. Kosztołowicz, Physica A 24844 (1998), J. Phys. A: Math. Gen. 311943 (1998).

11 J. Casas-Vásquez and D. Jou, Rep. Prog. Phys. 661937 (2003).

12 R. E. Lagos, T. P. Simões \& A. L. Godoy, Physica A 257 401 (1998). 